The use of higher stocking rates typical for short-duration grazing and an initially patchy distribution of legume and grass created very heavy selective pressure against the legumes, which were additionally stressed by the herbicide used to control sour dock. Dallisgrass and other weedy, summer-growing annual grasses were prominent invaders in the second year.

Both the single-probe capacitance meter and orchardgrass heights adequately followed forage availability changes over the 3-day grazing and 21- or 27-day regrowth periods. The probe was less useful in predicting required stocking rates and was less well related to animal liveweight gain than was the case for orchardgrass heights. It should be borne in mind that our monitoring methods differed from those used by others.
Where pasture entry forage levels are moderate to high, the 3-day utilization profiles suggest that stocking for a 2-or even 1day grazing period could be more efficient.

Kenneth L. Taggard is Plant Ecologist and Staff Research Associate, Charles A. Raguse is Professor and Agronomist, and Melvin R. George is Extension Range and Pasture Specialist, Department of Agronomy and Range Science; John L. (Roy) Hull is Specialist, and Cynthia A. Daley is Staff Research Associate, Department of Animal Science, University of California, Davis. John L. (Mike) Connor is Superintendent, Sierra Foothill Range Field Station. The authors appreciate the valuable assistance of Donald L. Springsteen and other members of the support staffat the station, and David F. Lile, Lab Assistant, Agronomy and Range Science, Davis.

\title{
Managing bovine pinkeye in beef calves
}

\author{
Lisle W. George
}

\section{Two field studies showed that a widespread pinkeye epidemic in a herd can be effectively treated with injections of long-acting oxytetra- cycline, followed by feeding the antibiotic in the ration. When the disease is less prevalent, injecting affected animals with penicillin or oxytetracycline is effective.}

Bovine pinkeye is one of the most economically important infectious diseases of beef cattle in the United States. A recent survey of 2,000 American cattle producers has shown pinkeye to be overwhelmingly considered the most troubling disease in their cattle. Annual losses to the disease in the United States have been estimated at $\$ 20$ million. Because of the ocular pain and the poor vision, calves do not eat properly and fail to thrive. One feedlot study demonstrated 260-day post-weaning depressions of 35 and 47 pounds in calves that had pinkeye in one or both eyes, respectively. Other pinkeye-related losses include the cost of antibiotics and the labor required to administer drug treatments.

During an outbreak of pinkeye, as many as $45 \%$ of yearling calves become infected and develop painful corneal ulceration. In approximately half of the cases, the ulcers enlarge, encompass the entire corneal sur- face and cause blindness. Most corneal ulcers usually heal by 45 days, but about $1 \%$ of them perforate, resulting in loss of the vitreous body and eventually producing a small, shrunken, nonfunctional eye. Blind calves are difficult to move and often are discriminated against when they are sold at auction.

Pinkeye is caused by the bacterium Moraxella bovis. The bacterium is highly susceptible to antibiotics and is easily eliminated from the eye tissues by three to four daily subconjunctival injections of penicillin. However, the disease often attacks a large number of susceptible animals within a herd and tends to recur frequently over the entire summer grazing season. Gathering a large number of affected calves from the pasture and injecting drugs is difficult. If done improperly, the treatment itself could damage the eye. Failure to disinfect the hands and equipment between animals could foster the transmission of the bacterium to normal eyes. Also, local ophthalmic treatment of affected cattle does nothing for the infected cattle without symptoms that act as reservoirs for the bacterial infection. Studies were therefore conducted to identify alternative methods of drug treatment for bovine pinkeye.

\section{Pinkeye treatment studies}

Initial studies investigated a long-acting formulation of oxytetracycline (LA-200). Calves were experimentally infected and then treated at the peak of clinical signs 7 to 14 days later. The treatment consisted of an intramuscular injection of the long-acting formulation into the muscles of the rear leg. The drug was administered at a dose of 20 milligrams per kilogram $(\mathrm{mg} / \mathrm{kg}$ ) of body weight. A second dose was administered 72 hours later.

This treatment was highly effective in eliminating the bacterial infection and reducing clinical signs. A later study showed that selective distribution of the oxytetracycline to the epithelium of the conjunctiva and the cornea was primarily responsible for its effectiveness against $M$. bovis.

During the summers of 1985 and 1986, severe pinkeye outbreaks occurred in the cattle at the Sierra Foothill station. This natural occurrence of the disease provided an opportunity to examine the use of oxytetracycline in a true field situation.

The first of two separate studies compared the effectiveness of two intramuscular injections of the long-acting oxytetracycline formulation with that of furazolidone topical spray (Topazone). The study was conducted between June 13 and August 6 .

At the beginning, 103 Hereford female calves ( 4 to 8 months of age) were assigned to one of three groups: oxytetracycline treated (OTC), furazolidone treated (FZ), and not treated (control). Treatments consisted of two applications $(20 \mathrm{mg} / \mathrm{kg}$ ) of long-acting oxytetracycline two times at a 72-hour interval (OTC group), and three daily applications of furazolidone spray (FZ) group. Treatments were not administered again unless the ocular lesions worsened, the ulcer healed and then recurred, or the opposite eye developed a corneal ulcer.

The calves were examined three times weekly for signs of ocular inflammation. At the time of each examination, a clinical severity score was assigned to each eye, and

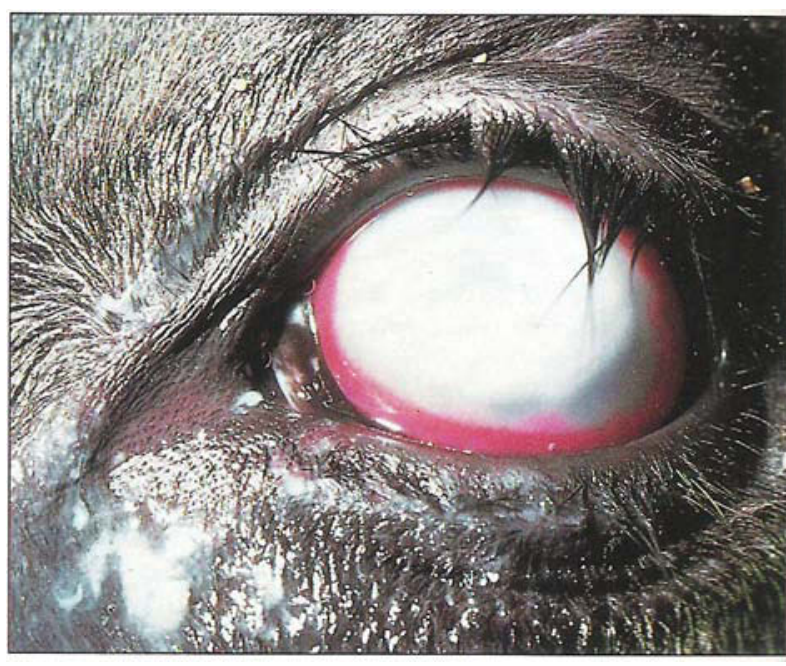

Pinkeye, an infectious bacterial disease in cattle, can cause a painful corneal ulcer that in some cases may lead to blindness. 
eyes with corneal lesions were photographed. The surface area of each corneal ulcer was measured from the photographic image. Secretions from all of the eyes also were cultured weekly to determine whether the antibiotic treatment successfully eliminated $M$. bovis. Calves were weighed weekly to determine the effects of the drug therapy on weight gains. At the end of the study, all $M$. bovis isolates were tested for susceptibility to the tetracyclines and the nitrofurans.

Corneal ulcers occurred in 102 calves. By the 22nd day of the study, fewer calves in the OTC group had corneal ulcers than in the other two groups. OTC calves had the most rapid healing rate and the lowest incidence of multiple ulcer recurrences. The rate of $M$. bovis isolation from the eyes of this group was significantly less than from the other two groups. Calves in the FZ group had more severe ocular lesions and a greater number of multiple ulcer recurrences than the OTC group calves, but were significantly better off than the controls. The $M$. bovis from the OTC group did not become resistant to either furazolidone or tetracycline, whereas the $M$. bovis from the FZ group calves showed a slight increase in resistance to the nitrofurans.

The average weekly body weights and weight gains were similar for the three treatment groups. Despite the clear therapeutic effectiveness of oxytetracycline over that of furazolidone, a cost analysis of the treatments (including the labor required to gather the cattle and administer the drugs) indicated that the OTC treatment had the highest cost of the three groups.

The second study compared oxytetracycline and penicillin treatments. Beginning on June 10, 119 calves were randomly assigned to one of three groups: 39 designated as OTC, 40 Pen, and 40 nontreated controls. The calves were kept in a common pasture during the entire summer and were examined three times daily as in the first study.

Treatments were administered to the calves of the OTC and Pen groups after an initial 4-day observation period. Affected calves of the OTC group were given two intramuscular injections of the long-acting oxytetracycline formulation $(20 \mathrm{mg} / \mathrm{kg}) 72$ hours apart. All of the OTC calves then were fed 2 grams per head per day of oxytetracycline in alfalfa pellets for 10 days. Calves of the Pen group that had corneal ulcers were treated with three daily subconjunctival injections of procaine penicillin $G$ (300,000 IU per injection). In both groups, treatments were administered to individual calves again if they developed a new corneal ulcer in either eye, or if the existing ulcer worsened. Calves of the control group remained untreated for the entire summer.

The prevalence of pinkeye was significantly reduced in the Pen and OTC groups within 12 days after treatment. OTC calves had significantly fewer ulcers than did calves of other two groups. Between days 16 and 44, the prevalence of pinkeye in the OTC calves ranged between 0 and 2 cases, whereas the penicillin-treated calves and controls ranged from 3 to 8 and 19 to 22 cases daily, respectively. Oxytetracycline and penicillin groups had significantly fewer active cases of pinkeye in individual calves than the controls. The two drugs were similar in effectiveness for the individual case. The healing times and average ulcer size for corresponding numbers of days after treatment were similar in the OTC and the Pen groups. The rate of $M$. bovis isolations from the eyes of the OTC group calves was significantly lower than that of the other two groups.

The results of the second study strongly suggest that a combination of "blitz" therapy with a long-acting formulation followed by short-term feeding of oxytetracycline effectively controlled an outbreak of pinkeye. Subconjunctival penicillin treatment, however, was as effective as the longacting formulation for treatment of individual cases of pinkeye.

A cost/benefit analysis was not performed in this study, but the OTC calves gained an average of 6 pounds more than the controls did and 3 pounds more than the Pen group. Whether this increase in weight gain was sufficient to offset the high purchase cost of the oxytetracycline is unclear.

\section{Conclusions}

The findings of these studies suggest that, whenever the prevalence of pinkeye is low (less than 10\%), the disease can be managed effectively by treating affected animals with three daily subconjunctival injections of procaine penicillin $\mathrm{G}$ ( $1 \mathrm{ml}$ per injection). If there is significant difficulty in administering the drug into the ocular tissues, intramuscular injection of a long-acting oxytetracycline formulation $(20 \mathrm{mg} / \mathrm{kg})$ in two doses given 72 hours a part is also effective.

In a widespread epidemic of the disease where the prevalence exceeds $10 \%$ to $20 \%$, or if it is desirable to minimize ocular scarring, the cattle should be "blitz" treated with long-acting oxytetracycline (two injections spaced 72 hours apart, $20 \mathrm{mg} / \mathrm{kg}$ per injection), and then fed oxytetracycline ( 2 grams per head daily for 10 days).

As with all infectious diseases, affected cattle should be removed from the clinically normal animals. Hands and equipment should be washed thoroughly in chlorhexidine solution after each affected animal is examined or treated.

Control of flies with insecticides delivered through backrubbers or dust bags is highly desirable.

Lisle W. George, DVM, Ph.D., is Associate Professor, Department of Medicine, College of Veterinary Medicine, University of California, Davis.

\title{
Supplements evaluated for wintering range calves
}

\author{
John R. Dunbar a Cindy A. Daley $\square \quad$ J.M. Connor \\ Charles B. Wilson $\square$ Charles A. Raguse $\square$ Thomas R. Famula \\ Melvin R. George
}

\section{In two range feeding trials to evalu- ate supplemental nitrogen and/or bypass protein source and stock- ing densities, calves at a low den- sity gained more weight than high- density groups. Dollar return dur- ing the supplementation phase, however, was highest from high- density groups fed a combination of urea and corn gluten meal.}

California annual rangeland forage is generally of poor quality in the fall and in short supply during the winter. During those periods, supplements are typically pro- vided to wintering calves to correct nutrient deficiencies and improve performance, maintain health, and prevent death loss.

Supplemental nutrients may be provided in many forms, the most common being hay, meals, pellets, cubes, liquids, and blocks. Supplements are usually high in nonprotein nitrogen such as urea, which aids fiber digestion and microbial growth. In many situations, however, urea supplementation may not meet the protein requirements of growing calves. Dietary protein may be digested to a variable degree in the rumen or may be entirely degraded. Protein that is not digested in the rumen, called bypass or escape protein, passes to the lower tract and is either digested postru- 